Archives

14-15 | 1995

Les banques de données au $\mathrm{CRH}$

\title{
Une banque de données d'images
}

\section{Perrine Mane}

\section{(apenEdition Journals}

\section{Édition électronique}

URL : http://journals.openedition.org/ccrh/2672

DOI : $10.4000 /$ ccrh. 2672

ISSN : $1760-7906$

\section{Éditeur}

Centre de recherches historiques - EHESS

\section{Édition imprimée}

Date de publication : 14 octobre 1995

ISSN : 0990-9141

\section{Référence électronique}

Perrine Mane, "Une banque de données d'images », Les Cahiers du Centre de Recherches Historiques [En ligne], 14-15 | 1995, mis en ligne le 27 février 2009, consulté le 26 avril 2019. URL : http:// journals.openedition.org/ccrh/2672; DOI : 10.4000/ccrh.2672

Ce document a été généré automatiquement le 26 avril 2019

Article L.111-1 du Code de la propriété intellectuelle. 


\title{
Une banque de données d'images
}

\author{
Perrine Mane
}

1 L'apport de l'iconographie pour l'étude de la culture matérielle est l'axe majeur de mes recherches, ce qui implique de gérer un nombre considérable de documents. Déjà lors de ma thèse de troisième cycle portant sur 127 calendriers monumentaux français et italiens des $\mathrm{XII}^{\mathrm{e}}$ et $\mathrm{XIII}^{\mathrm{e}}$ siècles - soit environ 1500 représentations si l'on tient compte des douze mois de l'année - avait nécessité la rentrée des données sur ordinateur pour effectuer tris ou statistiques.

2 Depuis cette époque, le nombre de documents s'est considérablement accru, le fonds s'étendant à plusieurs supports. La majorité provient de miniatures ou de gravures médiévales, mais comprend aussi d'autres techniques artistiques les unes relevant de l'art monumental, les autres dites mineures comme les tapisseries, les ivoires, les sceaux ou les émaux. Ces figurations datent $d u \mathrm{Ix}^{\mathrm{e}}$ au $\mathrm{Xv}^{\mathrm{e}}$ siècle et sont d'origine géographique très diverse.

3 La banque de données couvre aujourd'hui un corpus de 15000 enluminures provenant de 4000 manuscrits, de 1130 sculptures, 1700 peintures, 550 gravures et 700 divers, soit 19080 items.

4 Le but de la gestion informatique de cette iconothèque est de décrire et de classer plusieurs milliers d'images que la mémoire n'est plus capable de répertorier. Le choix de ce travail a été d'associer à chaque image un descriptif en mode texte. Les documents ont été répartis en cinq fichiers suivant le critère du support artistique : manuscrit, peinture, gravure, sculpture et un ensemble appelé divers, regroupant les autres techniques minoritaires, la tapisserie, le vitrail, la mosaïque, l'émail ou l'ivoire.

5 En vue de recherches ponctuelles (par exemple sortie des images contenant le mot clôture dans la rubrique architecture), des fichiers partiels peuvent être créés temporairement.

6 Le logiciel utilisé est DBase 3+ qui a été paramètré pour mes recherches personnelles ; de plus, faute de trouver un thesaurus répondant à mes besoins, il a été indispensable de constituer un nouveau système descriptif qui convienne à la relative homogénéité des images traitées et qui permette une saisie rapide. 
7 A chacune des images correspond un certain nombre de rubriques, relatives d'une part aux caractéristiques techniques de l'image, d'autre part à sa description.

8 Les caractéristiques techniques regroupent les champs suivants, par exemple pour les manuscrits : ville de conservation, bibliothèque, cote et fonds, folio, emplacement (marge, initiale...), auteur et titre de l'ouvrage, thème de l'enluminure, date d'exécution du manuscrit, origine géographique de l'enluminure, le miniaturiste, le destinataire du manuscrit, la référence textuelle de l'image, enfin le type de reproduction possédé.

9 Les champs concernant la description des images sont au nombre de onze: l'action exécutée, les outils figurés, la faune, les végétaux et les éléments naturels, l'alimentation (aliments et instruments de cuisine), le costume, le mobilier, l'architecture, les transports ou les récipients, les objets. Enfin une rubrique "divers" permet, lorsque la lecture d'une image n'est pas évidente, d'introduire des termes n'entrant pas dans les champs précédents ou même une légende très courte qui facilitent l'identification de l'image.

10 Il est vrai que les artistes pouvaient faire preuve de maladresse ou même d'ignorance, comme l'indiqueraient les nombreuses Apocalypse où la vendange des Nations s'effectue fréquemment avec la serpe à dos tranchant de la taille à moins que ce ne soit une faucille, outil commun aux vendanges et aux moissons des nations qui servent à couper les grappes. On retrouver ces confusions dans un livre d'heure où en octobre (Paris, BN, Lat 10533, f. 10.), le vigneron se sert d'une serpe à dos tranchant pour vendanger.

11 S'il est évident qu'associer des informations écrites à des documents figurés ne permet pas de décrire totalement une image et si une infinité de points de vue existe, la création de cette banque de données - répondant bien évidemment à mes recherches - permet toutefois de manipuler automatiquement d'importantes masses de données. Son association, je l'espère dans un avenir proche, avec un CD ROM, permettrait d'accéder encore plus facilement à un fonds d'images ingérable par la seule mémoire.

\section{Bibliographie :}

13 Traitements informatiques et iconographie, Le médiéviste et l'ordinateur, n 26-27, automne 1992-printemps 1993. 